\title{
Predictability of a low-order interactive ensemble
}

\author{
L. Siqueira and B. Kirtman \\ Rosenstiel School of Marine and Atmospheric Science, University of Miami, Miami, USA
}

Correspondence to: L. Siqueira (lsiqueira@rsmas.miami.edu)

Received: 30 June 2011 - Revised: 19 September 2011 - Accepted: 20 March 2012 - Published: 10 April 2012

\begin{abstract}
In this paper, numerical and analytical studies were performed to uncover the mechanisms controlling the changes in ensemble spread of a low-order coupled model with multiple atmospheric realizations. An interactive ensemble approach was applied to a coupled dynamical system based on two versions of the Lorenz 63 model designed in order to imitate the behavior of a coupled system with different time scales. In the dynamic system used in this work the spread of ensemble members is highly dependent on the mean state corresponding to asymmetries in predictability. The slowness of the slow model and the intensity of the boundary forcing anomalies both contribute to the asymmetry and phase locking of both subsystems. The mechanisms controlling the fast model spread were uncovered revealing uncertainty dynamics depending on the location of ensemble members in the fast model phase space and implicitly on the slowness and magnitude of the slow model anomalies.
\end{abstract}

\section{Introduction}

The atmospheric response or teleconnections associated with the El Niño-Southern Oscillation (ENSO) is found in both the tropics and extratropics. The predictability of the ENSO teleconnections is typically described in terms of whether the signal (usually measured by the ensemble mean) is large enough to exceed the climate noise (usually measured by the ensemble spread) due to internal dynamics. We simply argue that there is predictability when the signal-to-noise ratio is larger than one, e.g. Shukla et al. (2000), although it is possible to argue for predictability when the ratio is less than one.

Nevertheless, the predictability depends on relative amplitudes of the signal and, for our purpose here, the noise. If the amplitude of the noise is independent of the signal, then we can simply focus on the amplitude of the signal. Conversely, if the amplitude of the noise is affected by the signal then this dependence needs to be understood in our predictability assessments. Kirtman et al. (2005), for example, use the interactive ensemble coupling strategy (Kirtman and Shukla, 2002) to show that atmospheric wind stress uncertainty, i.e. spread, in their coupled general circulation model (CGCM) is larger for warm ENSO events. This would suggest that warm events are less predictable than cold events which seemed to be born out with retrospective predictions made with the same model. Wu and Kirtman (2006) also examined signal dependent noise amplitude in the same interactive ensemble simulation and found that cold events had smaller rainfall spread, i.e. noise, than warm events. Our focus here is to understand the source of the signal dependent noise amplitude from a dynamical systems perspective.

In this paper, numerical and analytical studies were performed to uncover the mechanisms controlling the changes in ensemble spread of a low-order coupled model with multiple atmospheric realizations. Motivation for this study comes from a related issue whether climate anomalies are more predictable in El-Niño years than in La-Niña years. If that turns out to be the case, it promptly gives rise to another questioning: what mechanism could bring the asymmetry in predictability between the two states?

This question can be addressed in the context of ensemble simulations of atmospheric general circulation models (AGCMs). In ensemble simulations the noise (atmospheric internal variability) is measured by the ensemble spread and the signal is represented by the ensemble mean. If one assumes that the climate variability is described by a linear damped dynamic system (Hasselman, 1976; Frankignoul and Hasselman, 1977), then the interannual variation in the amplitude of noise cannot be related to that in the signal (state of the system). This is, perhaps, conceptually clear given that the noise is externally prescribed in this conceptual model. There are indications in the literature that this relationship does not hold. Kirtman et al. (2005) suggest that the spread in the zonal wind stress is highly dependent on the 
ensemble mean with small spread for cold ENSO events and large spread for warm ENSO events in the CGCM studied. Wu and Kirtman (2006) analyses found that the predictability for the equatorial central Pacific rainfall is much higher in $\mathrm{La}$ Niña years than in El Niño years, whereas the predictability for 500-hPa height in the western tropical Pacific-Australian region is higher in El Niño years than in La Niña years. Thus, the changes in predictability are expected to depend on the model employed as well as on which variable is being analyzed. Our focus here is to understand the source of the signal dependent noise amplitude from a dynamical system perspective.

Here we employed the same coupling strategy (Kirtman and Shukla, 2002) that has been developed to examine the relative importance of stochastic forcing and deterministic coupling in generating climate variability in CGCMs. However, in this work we brought this strategy for the coupling between two low-order models with different time scales. Low-order models originally conceived as means of illustrating some of the effects of nonlinearity have proven useful in investigating specific problems, some of which could not readily be studied in complex systems like modern CGCMs. We made use of numerical simulations and an analytic approach in order to reveal the uncertainty dynamics leading to changes in ensemble spread and the mechanism for the asymmetry in predictability between the two states.

\section{Model, results and uncertainty dynamics}

The predictability of a particular dynamical system can be studied by investigating the evolution of finite uncertainties over some prescribed interval of time or by performing ensemble simulations and monitoring the spread between ensemble members. In the context of ensemble simulations low-spread periods are essentially more predictable than high-spread periods. In terms of dynamics of small uncertainties the time interval used to define growth rate can be of different durations ranging from infinitesimal to yield an instantaneous growth rate, finite yielding an "effective" growth rate, or infinite yielding global measures like maximum Lyapunov exponents (MLE). The predictability limit of a particular system can be estimated in terms of these time scales which indicates when the forecast uncertainty exceeds some limit or when information of the initial condition is lost. It is held that the predictability limit is eventually related to the inverse of the MLE (Boffetta et al., 1998). However, the inhomogeneity common in nonlinear chaotic systems limits the application of global measures like the MLE. In this work, both uncertainty dynamics and ensemble simulations are used to investigate the predictability fluctuations of a low-order coupled model.

The Lorenz model (Lorenz, 1963) has been widely used as a conceptual model for predictability studies (Tsonis, 1992; Palmer, 1993) and provides a practical test case with in- teresting qualitatively properties. Atmospheric behavior involving barotropic and baroclinic instabilities is considered somewhat analogous to Lorenz 63 model behavior because of the exponential instability of the model's trajectories and its abrupt regime changes (Miller et al., 1994). However, the predictability limit associated with timescales of evolving uncertainties is a model intrinsic quantity, therefore generalization between different nonlinear systems can be difficult.

We first formulate a coupled dynamical system where two versions of the Lorenz 63 model were coupled in order to imitate the behavior of a coupled system with different time scales (Boffetta et al., 1998; Peña and Kalnay, 2004). This coupling scheme includes coupled feedbacks analogous to the tropical atmosphere/ocean, although in this work the model used is not physically-based, it does have the property of two coupled systems with very different time scales. To convert an ODE to a similar ODE that runs slower, we start with the required form in a time variable $t^{\prime}$

$$
\frac{d \mathbf{x}}{d t^{\prime}}=\mathbf{f}(\mathbf{x}, \mathbf{y})
$$

where the vector field includes dependency on variables of a coupled system, and apply a time scale $t^{\prime} \rightarrow \tau t, \tau<1$, to obtain

$\frac{1}{\tau} \frac{d \mathbf{x}}{d t}=\mathbf{f}(\mathbf{x}, \mathbf{y})$

hence:

$\frac{d \mathbf{x}}{d t}=\tau \mathbf{f}(\mathbf{x}, \mathbf{y})$.

Consider a fairly general expression of the coupled Lorenz 63 system, where the slow subsystem variables $\left(x_{o}, y_{o}, z_{o}\right)$ have a different intrinsic time scale. For the fast model

$$
\begin{aligned}
\frac{d x_{a}}{d t} & =\sigma\left(y_{a}-x_{a}\right)-a_{1}\left(x_{o}+k_{1}\right) \\
\frac{d y_{a}}{d t} & =r x_{a}-y_{a}-x_{a} z_{a}+a_{1}\left(y_{o}+k_{1}\right) \\
\frac{d z_{a}}{d t} & =x_{a} y_{a}-b z_{a}+a_{1} z_{o}
\end{aligned}
$$

and for the slow model

$$
\begin{aligned}
\frac{d x_{o}}{d t} & =\tau\left[\sigma\left(y_{o}-x_{o}\right)-a_{2}\left(x_{a}+k_{2}\right)\right] \\
\frac{d y_{o}}{d t} & =\tau\left[r x_{o}-\tau y_{o}-\tau x_{o} z_{o}+a_{2}\left(y_{a}+k_{2}\right)\right] \\
\frac{d z_{o}}{d t} & =\tau\left[x_{o} y_{o}-\tau b z_{o}-a_{2} z_{a}\right]
\end{aligned}
$$

where $\sigma=10, b=8 / 3$, and $r=28$ are the standard values of the Lorenz 63 model parameters. The following parametrization is used in order for the coupled model qualitatively capture the ENSO time-scales

$a_{1}=\alpha, \quad a_{2}=\frac{\alpha}{\tau}, \quad k_{2}=\frac{k_{1}}{\tau}$ 
Table 1. Model parameters in Eq. (4) to Eq. (9).

\begin{tabular}{cc}
\hline Parameter & Value \\
\hline$\sigma$ & 10 \\
$\mathrm{~b}$ & $8 / 3$ \\
$\mathrm{r}$ & 28 \\
$\tau$ & 0.1 \\
$\alpha$ & 0.8 \\
$k_{1}$ and $k_{2}$ & -10 \\
\hline
\end{tabular}

where $\alpha$ is the coupling strength, and $k_{1}$ is an offset parameter taken to be -10 . When $\alpha=0$ the original Lorenz 63 model is recovered. The different time scales are set through a temporal scale factor where $\tau=0.1$ implies that the slow model is 10 times slower than the fast model. Table 1 summarizes the parameters used in the fast-slow Lorenz model in order for the model qualitatively capture the ENSO time-scales.

The original Lorenz 63 model shows random reversals of the streamfunction and horizontal temperature gradient. In the absence of any imposed forcing, the probability density functions (PDFs) associated with the two different regimes (wings) of the original Lorenz 63 model are equal. The offset parameters $k_{1}$ and $k_{2}$, only acting over $x$ and $y$, play the role of an imposed forcing to ensure that the system resides in the "neutral-cold" state more frequently than the warm state.

In the toy model, the fast system streamfunction $x_{a}>0$ (clockwise circulation) enhances $x_{o}<0$ (counterclokwise) for neutral-cold conditions, while $x_{a}<0$ (counterclokwise) enhances $x_{o}>0$ (clockwise circulation) for warm conditions. This is why there is a negative sign in the coupling. Despite the negative sign in the coupling through the $x$ variables, it is actually a positive feedback since the opposite circulations work to enhance each other. The $y$ variable is related to the horizontal temperature gradient and also acts as a positive feedback since the temperature gradient in one subsystem enhances the temperature gradient in the same direction in the other subsystem, therefore the positive sign.

The positive feedback, by itself, would lead to an instability that would either "lock" the system into a permanent cold or warm state much like the Bjerknes feedback. The coupling terms involving $x$ and $y$ variables are positive feedbacks, therefore, if these were the only mechanisms at work the system would never flip from one state to the other. To include the damping that limits the growth of instabilities in the slow system, the coupling through the $z$ variable (vertical temperature deviation) acts to increase $z_{a}$ when there is a warm anomaly in the slow model, while a positive anomaly in $z_{a}$ acts to dampen the slow system (negative feedback). Therefore, the coupling between the two subsystem is asymmetric.

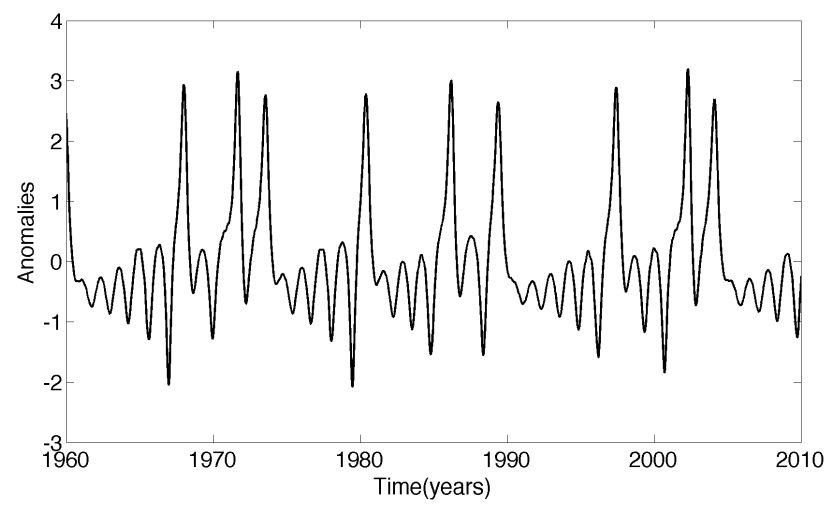

Fig. 1. Time series of slow model $x_{o}(t)$ anomalies.

The oscillation between states in the toy model is only due to the instability of the fixed points in the chaotic regime. However, the model will prove to be a useful conceptual tool. It will facilitate an investigation of some dynamical features and can provide analogues for the behavior of more complicated systems. Moreover, we can develop an analysis tool that can be generalized to CGCMs. In certain ways the model imitates a coupled CGCM: it is forced, dissipative, and chaotic. The "atmosphere" is more rapidly-evolving than the "ocean", and "air-sea" interactions produce variability with multiple time scales in both components. The lower boundary conditions provided by the slow model are slowlyevolving (much like sea surface temperatures) and can lend partial predictability to the fast model. The relative simplicity of the low order coupled model is a suitable characteristic to perform predictability analysis since it allows the use of a large number of ensemble members with extended runs.

Equations were integrated using a fourth order RungeKutta time scheme. The initial transient period is discarded. Numerical integration indicate that trajectories $\mathbf{x}(t)=\left(x_{a}, y_{a}, z_{a}, x_{o}, y_{o}, z_{o}\right)^{T}$ of the coupled model remain within a bounded region (attractor), but at the same time depend sensitively on the initial state. Numerical simulations with different coupling parameters were performed in order to identify a suitable parameter range for this model. The choice of parameters for the control run was made in order to qualitative mimic the time series for NINO3.4 SST anomalies (Fig. 1). This is achieved when the coupling parameter is set to $\alpha=0.8$ since with this coupling strength the model qualitatively imitates the observed variability, and the longterm behavior of the coupled dynamics shows an intricate interaction between periodicity and randomness. Figure 1 shows the slow model $x_{o}(t)$ anomalies. The slow "ocean" is fluctuating between a "neutral-cold" state which lasts typically 3 to 7 "years" and a warm state which lasts only one "year".

Lyapunov exponents and phase locking play an essential role in the analysis of synchronization with coupled chaotic systems (Duane and Tribbia, 2007) and are employed in this 
work. In this study the phase of both subsystems are extracted via Hilbert transform in order to determine phase locking intervals and synchronization at different coupling strengths. The phase of a time series $x(t)$ can be determined by using the analytic signal approach (Rosenblum et al., 1996) where the analytic signal $\tilde{x}(t)$ is a complex function of time

$Z_{x}(t)=x(t)+i \tilde{x}(t)=A_{x}^{H} e^{i \Phi_{x}^{H}(t)}$

and $\tilde{x}(t)$ is the Hilbert transform of $x(t)$

$\tilde{x}(t)=\frac{1}{\pi} \mathrm{P} \cdot \mathrm{V} \cdot \int_{-\infty}^{+\infty} \frac{x\left(t^{\prime}\right)}{t-t^{\prime}} d t^{\prime}$

(P.V. means that the integral is taken in the sense of the Cauchy principal value). The instantaneous Hilbert phase is given by:

$\Phi_{x}^{H}(t)=\arctan \frac{\tilde{x}(t)}{x(t)}$.

For each of the two phase distributions a phase locking index is computed. The index for measuring phase synchronization is given by:

$\gamma^{H}=\left|\frac{1}{N} \sum_{j=1}^{N} e^{i\left[\Phi_{x}\left(t_{j}\right)-\Phi_{y}\left(t_{j}\right)\right]}\right|$.

For this model, the whole system is six dimensional with two positive, two zero and two negative Lyapunov exponents. As the coupling strength $(\alpha)$ is increased all the exponents evolve revealing stages of synchronization, therefore the exponents for coupled chaotic systems are also a function of coupling strength. Figure 2 shows $\gamma^{H}$ (mean phase coherence), and the two maximum Lyapunov exponents for each component as functions of the coupling strength. For coupling strengths $\alpha \gtrsim 0.9$ the originally positive Lyapunov exponent of the fast model attains negative values, indicating that generalized synchronization between the systems takes place. For stronger coupling $\gamma^{H}$ rises to values closer to 1 , finally leading to identical synchronization for higher coupling strenghts.

When coupling strength is set to $\alpha \gtrsim 1.2$ all six conditional Lyapunov exponents become negative and both subsystems approach a limit cycle eventually reaching equilibirum state for larger $\alpha$. For coupling strengths $0.6 \lesssim \alpha \lesssim 1.2$ the slow model $\lambda_{+}$suggests that it is mainly behaving as the driver $\left(\lambda_{+}^{\text {slow }}>0\right)$, while the fast model is behaving as the responder since its $\lambda_{+}^{\text {fast }}$ approaches zero for these coupling strengths. These values correspond to long term averaged Lyapunov exponents over the attractors which can be interpreted as an almost "slave" fast model with small amplitude, and regime changes modulated by the slow model. The coupling strength for the control run $(\alpha=0.8)$ was initially picked in order to qualitatively mimic the observed NINO3.4 time series which is located just before $\lambda_{+}^{\text {fast }}$ goes negative, $\lambda_{+}^{\text {slow }}$ still positive,

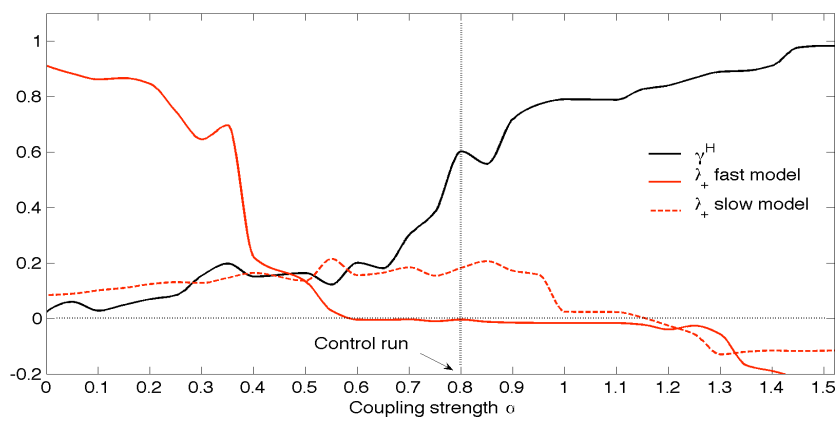

Fig. 2. Mean phase coherence $\left(\gamma^{H}\right)$ in black and conditional Lyapunov exponents $\left(\lambda_{+}^{\text {fast }}\right.$ and $\lambda_{+}^{\text {slow }}$ ) as a function of coupling strength $\alpha$. Fast model Lyapunov exponent in solid red and slow model lyapunov exponent in dashed red. (a)

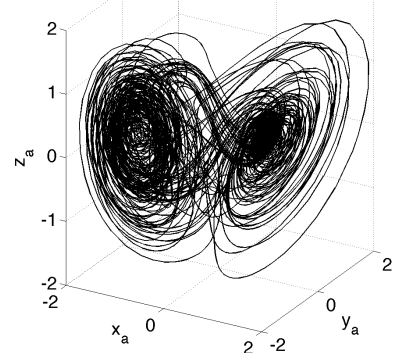

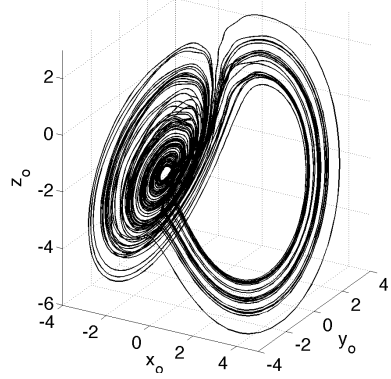

Fig. 3. Lorenz coupled system phase portrait. (a) fast subsystem; (b) slow subsystem.

and there is some degree of synchronization. This setting bears some resemblance to the model of Zebiak and Cane (1987), a model with a noise-free slave atmosphere.

The phase space of both subsystems is shown in Fig. 3, and the resulting attractor for both slow and fast components are quite different, the latter being less organized than the original Lorenz 63 model. Figure 3b shows the slow "ocean", vacillating between a more frequent regime "neutral-cold" and a less frequent warm state.

The coupling terms in each equation for the dynamics are equivalent to a variable forcing. For the Lorenz 63 model in the absence of forcing, both regimes of the attractor are equally probable. In this particular model the phase space position of the regime centroids does not change as the forcing rotates and change in intensity. However, when forcing is introduced in the $X-Y$ plane the probability of the model state lying in one of the regimes becomes greater than that in the other regime. These changes are due to the projection of the forcing along the dominant EOF of the system, which points between the two regime centroids (Palmer, 1993).

For the reduced Rayleigh number $r>24.75$, the equilibrium points $C^{+}\left(C^{-}\right)$which were unstable in the absence of forcing, can be made stable by sufficiently large 

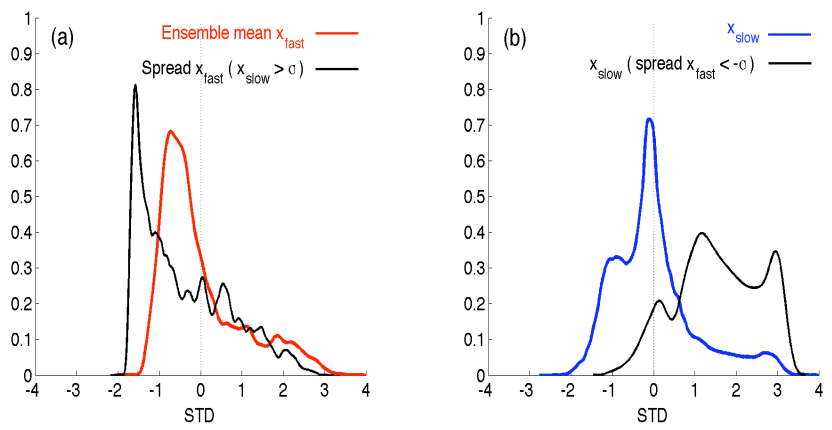

Fig. 4. (a) Pdf for the $x_{\text {fast }}$ anomalies from the interactive ensemble as a function of its standard deviation (STD) around the mean: ensemble mean in red and conditional ensemble spread - when $x_{\text {slow }}$ is greater than one standard deviation $(\sigma)$ of the slow model anomalies - in black; (b) Pdf for the $x_{\text {slow }}$ anomalies as a function of its standard deviation (blue) and conditional $x_{\text {slow }}$ - when spread of $x_{\text {fast }}$ is less than one standard deviation $(\sigma)$ of the fast model spread - in black.

positive (negative) forcing (Mittal et al., 2005). All these complex effects are acting simultaneously as the forcing changes with time in the coupled system.

The ensemble run performed in this study is different from a traditional coupled model for which individual atmospheric realizations are coupled to individual oceanic realizations. In the interactive ensemble coupling, multiple realizations of the fast model are coupled to one realization of the slow model (Kirtman and Shukla, 2002). The fast model realizations only differ in terms of their initial conditions. Each fast model realization experiences the same forcing produced by the slow model. The slow model is subjected to the ensemble average of fluxes from the fast model realizations. This is the interactive ensemble (IE) approach first introduced by Kirtman and Shukla (2002) applied to a simple coupled model.

In this IE coupling, the use of multiple fast model realizations allows the separation of the fast model variability due to boundary forcing (signal) and that due to internal dynamics. The ensemble mean for the fast model realizations is regarded as a lower boundary condition forced signal and the difference among the members (ensemble spread) is considered as noise generated by internal dynamics.

Extended runs with up to 100 ensemble members were performed and the probability density function (PDF) for the ensemble mean and ensemble spread are shown in Fig. 4 as a function of its standard deviation (STD) around the mean. For the fast model both the ensemble mean state and conditional ensemble spread - when $x_{\text {slow }}$ is greater than one standard deviation $(\sigma)$ of the slow model anomalies - are skewed in Fig. 4a. The conditional spread PDF indicates that when there are strong positive anomalies in the $x_{\text {slow }}$ variable, the spread is typically small (but not negative), i.e. the fast model uncertainty is relatively small (black line in Fig. 4a). This is typically a strong nonlinear system. For completeness,

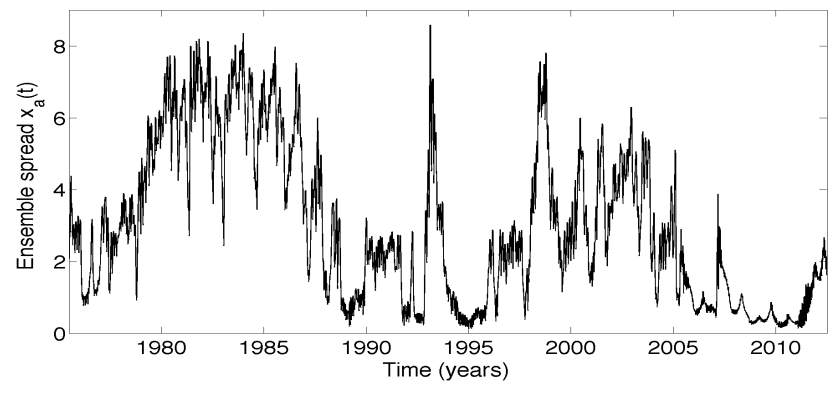

Fig. 5. Spread of $x_{a}$ ensemble members with no offset forcing $\left(k_{1}=\right.$ $\left.k_{2}=0\right)$.

the conditional $x_{\text {slow }}$ PDF - when the spread of $x_{\text {fast }}$ is less than one standard deviation $(\sigma)$ of the fast model spread is shown in Fig. 4b in black, and in this case small spread implies positive anomalies in the slow model. The asymmetry in predictability comes from the fact that the ensemble spread is strongly affected by a change in the state of the slow model. The slow model PDF (Fig. 4b) is skewed towards negative anomalies (blue). Its worth noting that both the $x_{\text {fast }}$ and $x_{\text {slow }}$ variables are skewed due to forcing through both the offset parameter and the coupling terms. The asymmetry in predictability is not dependent on the existence of the offset, as shown in Fig. 5 where the offset parameter was turned off.

That can be further explored if we consider that although both the offset and coupled feedbacks were intentionally set to qualitatively mimic the observations, one could imagine that for a linear model (linear propagator) the eigenvalues have fixed values for all points in phase space, thus the growth rate of uncertainties is the same no matter which is the state of the system. However, for nonlinear deterministic system or linear stochastically perturbed dynamics with multiplicative noise, a characterization of predictability for a system represented by a given marginal (that is, unconditional) PDF depends on the dynamics of the underlying system and cannot be inferred solely from the non-Gaussianity of the marginal PDF, as shown by Sura et al. (2005). Therefore, the inclusion of the offset in the toy model does not produce any asymmetry in predictability by itself.

Figure 6 shows the reason for the asymmetry in predictability which is explained by the ensemble spread being strongly affected by a change in the state of the slow model (ocean) from the "neutral-cold" state to warm state. On the top panel of Fig. 6 the ensemble spread is significantly reduced when a positive anomaly occurs. This period of increased predictability last until the slow model returns to "neutral-cold" conditions and can even persist into the neutral state for a period. This suggests that the slow "ocean" is reducing the "atmospheric noise" during some periods where there is higher predictability (reduced spread). What mechanism could be affecting the atmospheric spread in this coupled model? 


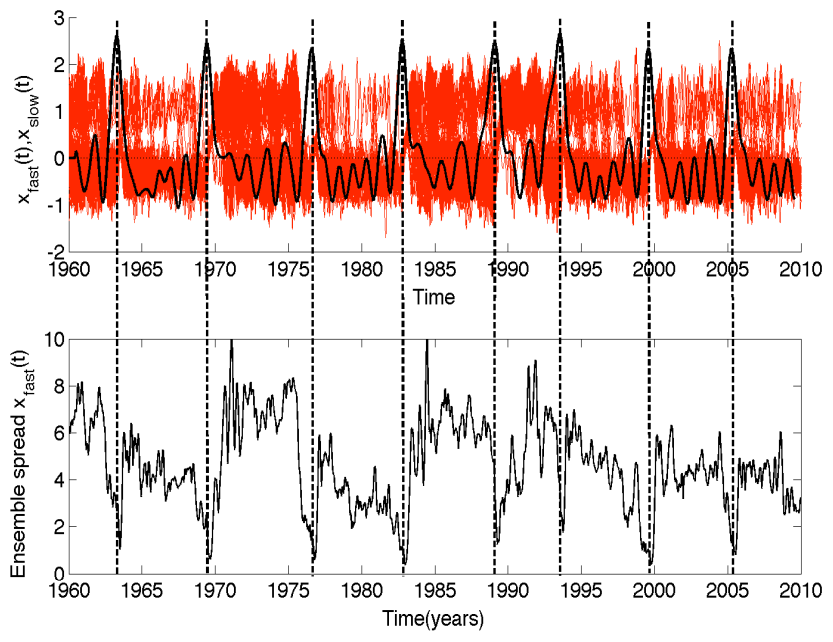

Fig. 6. Top: $x_{a}$ anomalies of ensemble members (red) and $x_{o}$ anomalies (black) time series. Bottom: Spread of $x_{a}$ ensemble members.

An analytic approach to this question, similar to Wittenberg and Anderson (1998), is possible by formulating the whole system as

$$
\frac{d \mathbf{X}}{d t}=N_{\mathbf{X}} \mathbf{X}
$$

where $N_{\mathbf{X}}$ is a nonlinear operator at the state vector $\mathbf{X}$. Since Eq. (15) represents a coupled system with linear coupling, then $N_{\mathbf{X}}$ consists of an uncoupled nonlinear part and an airsea interaction part

$\frac{d \mathbf{X}}{d t}=(U \mathbf{X}+C) \mathbf{X}$

where

$U_{\mathbf{X}}=\left(\begin{array}{cc}A_{\mathbf{X}} & 0 \\ 0 & O_{\mathbf{X}}\end{array}\right)$ and $C=\left(\begin{array}{cc}0 & C^{O \rightarrow A} \\ C^{A \rightarrow O} & 0\end{array}\right)$

$A_{\mathbf{X}}$ and $O_{\mathbf{X}}$ are the atmosphere and ocean uncoupled nonlinear operator, $C^{O \rightarrow A}$ and $C^{A \rightarrow O}$ are the sea-to-air interaction, and the air-to-sea interaction respectively (see the Appendix). We are interested in deriving an equation for the time evolution of the separation among ensemble members in the context of the interactive ensemble approach. We are considering a perfect model approach where the uncertainties come from small differences in the fast model initial conditions among different ensemble members. This can be done by defining the difference vector between two fast model states, $i$-th ensemble member $\mathbf{X}_{i}^{a}$ and a reference trajectory $\mathbf{X}_{j}^{a}$, so that the fast model uncertainty $\delta_{i}^{a}$ can be defined as deviations from the reference trajectory:

$\delta_{i}^{a}=\mathbf{X}_{i}^{a}-\mathbf{X}_{j}^{a} \quad \therefore \quad \mathbf{X}_{i}^{a}=\mathbf{X}_{j}^{a}+\delta_{i}^{a}$.

If the vector function $U_{\mathbf{X}} \mathbf{X}$ is continuous and differentiable everywhere, we can expand the solution around the reference trajectory $\mathbf{X}_{j}^{a}$ in powers of the difference $\delta^{a}$ (omitting the $i$ th ensemble member notation from here on) which gives the equation for ensemble member $\mathbf{X}_{i}^{a}$

$$
\begin{aligned}
\frac{d\left(\mathbf{X}_{j}^{a}+\delta_{a}\right)}{d t}=\frac{d \mathbf{X}_{i}^{a}}{d t}=A_{\mathbf{X}_{j}^{a}} \mathbf{X}_{j}^{a} & +A_{\mathbf{X}_{j}^{a}}^{\prime} \delta_{a} \\
& +O\left(\delta_{a}^{2}\right)+C^{O \rightarrow A} \mathbf{X}^{o}
\end{aligned}
$$

where $O\left(\delta_{a}^{2}\right)$ denotes nonlinear error terms obtained from the power series expansion of $A_{\mathbf{X}_{j}^{a}} \mathbf{X}_{j}^{a}, A_{\mathbf{X}_{j}^{a}}^{\prime}$ is the Jacobian of the vector function $A_{\mathbf{X}_{j}^{a}} \mathbf{X}_{j}^{a}$, and $\mathbf{X}^{o}=\left(x_{o}, y_{o}, z_{o}\right)^{T}$ are the slow model variables. It is worth noting that in the interactive ensemble approach all ensemble members experience the same surface boundary conditions (linear coupling term) $C^{O \rightarrow A} \mathbf{X}^{o}$ coming from the slow model. Subtracting from the equation for $\mathbf{X}_{i}^{a}$, i.e. Eq. (19), the following equation for the reference trajectory

$\frac{d \mathbf{X}_{j}^{a}}{d t}=A_{\mathbf{X}_{j}^{a}} \mathbf{X}_{j}^{a}+C^{O \rightarrow A} \mathbf{X}^{o}$

yields:

$\frac{d \delta_{a}}{d t}=A_{\mathbf{X}_{j}^{a}}^{\prime} \delta_{a}+O\left(\delta_{a}^{2}\right)$.

The nonlinear error terms $O\left(\delta_{a}^{2}\right)$ can be ignored when they are small relatively to $A_{\mathbf{X}_{j}^{a}}^{\prime} \delta_{a}$. The dynamics of a small fast model uncertainty is therefore governed by the linearization of the flow and the last equation provides a dynamical system for small uncertainties. It is worth noting that the coupling term was cancelled as expected since the fast model internal variability is, by definition, separated from the coupled signal.

However, in a nonlinear system the internal variability is not independent of the fast model mean state which in turn is affected by the slow model state, as previously shown in the PDF analysis. This implies that the coupling is important despite the absence of any coupling terms in Eq. (21). The coupled signal is participating implicitly by affecting the mean state of the fast model which in turn changes $A_{\mathbf{X}_{j}^{a}}^{\prime} \delta_{a}$, the Jacobian of the vector function $A_{\mathbf{X}_{j}^{a}} \mathbf{X}_{j}^{a}$. The vector function and its Jacobian depend on the time-evolving basic flow (time dependent propagators) and assume different values as the fast model visits different regions of the phase space. The coupled signal and the internal dynamics are the mechanisms governing the attractor's behavior of both fast and slow models and indirectly affect the growth of uncertainties. Therefore, the key to understand the uncertainty dynamics in this model is in estimating the effects of local instabilities in regions of the fast model phase space through which the ensemble trajectories are likely to pass.

We proceed to analyze which mechanisms can produce changes on the separation among ensemble members guided by Smith et al. (1999), who stressed the importance of the non-normality of the Jacobian matrix when computing 
growth rates. The instantaneous growth rate $r$ at time $t$ of an uncertainty is

$$
\begin{aligned}
r=\left.\frac{1}{\|\delta\|} \frac{d\|\delta\|}{d t}\right|_{t} & =\left.\frac{1}{\|\delta\|} \frac{d}{d t} \sqrt{\delta^{T} \delta}\right|_{t} \\
& =\frac{1}{2\|\delta\|^{2}}\left(\left.\frac{d \delta^{T}}{d t}\right|_{t} \delta(t)+\left.\delta(t)^{T} \frac{d \delta}{d t}\right|_{t}\right)
\end{aligned}
$$

where the superscript $T$ is the transpose operator and $\|\delta\|$ is the Euclidean norm expressed as square root of the inner product of the vector and itself. We can write the eigenvalue decomposition of $A_{\mathbf{X}^{a}}^{\prime}$ as $A_{\mathbf{X}^{a}}^{\prime}=\mathbf{E} \Lambda \mathbf{E}^{-1}$ considering that the matrices have distinct eigenvalues, the diagonal matrix $\Lambda$ contains the eigenvalues $\lambda_{i}$ of $A_{\mathbf{X}}^{\prime}$, and $\mathbf{E}$ its eigenvectors. The changes in $\delta$ will depend on: the eigenvalues of the Jacobian $A_{\mathbf{X}^{a}}^{\prime}$ and implicitly the background state, the projections of the uncertainty onto the eigenvectors $\delta^{T} \mathbf{E}$, and the decomposition of $\delta$ into the eigenvector $\mathbf{E}^{-1} \delta$.

If we turn our attention towards the eigenvalues of $A_{\mathbf{X}^{a}}^{\prime}$, the divergent directions along which the projection of $\delta_{a}$ grows equate to eigenvalues with positive real parts. Conversely, the convergent directions, along which the projection of $\delta_{a}$ shrinks, correspond to eigenvalues with negative real parts. However, as Smith et al. (1999) pointed out, the eigenvalues of $A_{\mathbf{X}^{a}}^{\prime}$ alone do not supply sufficient conditions to determine the sign of the growth rate since, in general, the eigen-basis is not orthogonal, i.e. $A_{\mathbf{X}^{a}}^{\prime}$ is non-normal.

Combining equations for the time evolution of the uncertainties Eq. (21) and the instantaneous growth rate Eq. (22) yields:

$r\left(\delta, \mathbf{X}^{a}, t\right)=\frac{\delta^{T}\left(A_{\mathbf{X}^{a}}^{\prime}+A_{\mathbf{X}^{a}}^{\prime}\right) \delta}{2 \delta^{T} \delta}$.

For this coupled model:

$\left(A_{\mathbf{X}^{a}}^{\prime}+A_{\mathbf{X}^{a}}^{\prime T}\right)=\left(\begin{array}{ccc}-2 \sigma & \sigma+r-z_{a} & y_{a} \\ \sigma+r-z_{a} & -2 & 0 \\ y_{a} & 0 & -2 b\end{array}\right)$.

Since $\left(A_{\mathbf{X}^{a}}^{\prime}+A_{\mathbf{X}^{a}}^{\prime}\right)$ is a symmetric matrix, it has orthogonal eigenvectors and its eigenvalues are real. In particular, if all eigenvalues of $\left(A_{\mathbf{X}^{a}}^{\prime}+A_{\mathbf{X}^{a}}^{\prime}\right)$ are positive (negative), $r\left(\delta, \mathbf{X}^{a}, t\right)$ will be positive (negative) independent of the orientation of the uncertainty. This symmetric matrix can be negative definite for sufficient model states. However, there is no state $\mathbf{X}^{a}=\left(x_{a}, y_{a}, z_{a}\right)^{T}$ in the fast model phase space where this matrix can be positive definite.

The eigenvalues analysis of $A_{\mathbf{X}^{a}}^{\prime},\left(A_{\mathbf{X}^{a}}^{\prime}+A_{\mathbf{X}^{a}}^{\prime}\right)$ and the implicit participation of the coupling through the fast model mean state provide the mechanisms controlling the growth rate of uncertainties in this model. This provides a conceptual picture explaining the behavior of the ensemble members. Whenever the ensemble members trajectories visit regions of phase space where all directions are convergent, the small uncertainties $\delta_{a}$ shrinks exponentially. Once back outside the convergent region, the uncertainties grow exponentially along divergent directions leading to an increase in the ensemble spread.

We proceed to locate regions in the fast model phase space where all instantaneous growth rates are negative and therefore all uncertainties must decrease for as long as the ensemble trajectories remain within those regions. Such regions must display enhanced predictability for finite times and coincide with the reduced ensemble spread periods shown in Fig. 6.

The eigenvalues of $A_{\mathbf{X}^{a}}^{\prime}$ and $\left(A_{\mathbf{X}^{a}}^{\prime}+A_{\mathbf{X}^{a}}^{\prime}\right)$ are obtained through the roots of the characteristic polynomial of each of these matrices evaluated at every point of the phase space. Regions with enhanced predictability are comprised of points with eigenvalues having negative real parts. There are important criteria that give necessary and sufficient conditions for all the roots of the characteristic polynomial with real coefficients having negative real parts which are known as RouthHurwitz Criteria (see the Appendix). This helps delineate the boundaries of the convergent subsets where all eigenvalues have negative real part. We follow the derivation of the explicit formulas for the surfaces separating these regions as in Smith et al. (1999) (see the Appendix).

We denote $V_{1}$ as a region where the Jacobian $A_{\mathbf{X}^{a}}^{\prime}$, at each point in phase space, have eigenvalues with negative real parts. For non-normal $A_{\mathbf{X}^{a}}^{\prime}$, this is not sufficient to rule out possible positive growth rates, yet such regions are, at least numerically, dominated by decreasing uncertainties with time. If, on the other hand, each eigenvalue of $\left(A_{\mathbf{X}^{a}}^{\prime}+A_{\mathbf{X}^{a}}^{\prime}\right)$ is negative within a region $V_{2}$, then no instantaneous growth rate within $V_{2}$ is positive. Therefore, all uncertainties will decrease with time for at least as long as the trajectories remain within $V_{2}$ and $V_{1}$. In general, $V_{2} \in V_{1} \in R^{m}$.

The coupled Lorenz system has a nonempty region $V_{1}$ and $V_{2}$ within which all uncertainties decay. The numerical simulation using the interactive ensemble approach confirm that these regions are dominated by decreasing uncertainty. Figure 7 shows the relative position of $V_{1}\left(V_{2}\right.$ not shown) to the attractor and the fast model ensemble members trajectories (yellow lines). The surface separates the $V_{1}$ region located above the blue side of the surface where all eigenvalues have negative real parts (convergent) and the region below the red side of the surface corresponds to the region where uncertainties grow exponentially along divergent directions.

We may also visualize the crossing of ensemble members trajectories into the $V_{1}$ subset, the subsequent locking to the ensemble mean, and synchronization with the ocean forcing by plotting the phase pairs $\Phi_{\text {slow }}$ (slow model) against $\Phi_{\text {fast }}$ (fast model) in Fig. 8. In asynchronous states the phase pairs $\left(\Phi_{\text {fast }}, \Phi_{\text {slow }}\right)$ fill most homogeneously the sub-plane interval and then make the phases statistically independent. Trajectories in the middle of Fig. 8 are in the divergent region (and asynchronous state) where the uncertainties grow exponentially (slow model is in neutral-cold state). In synchronized 


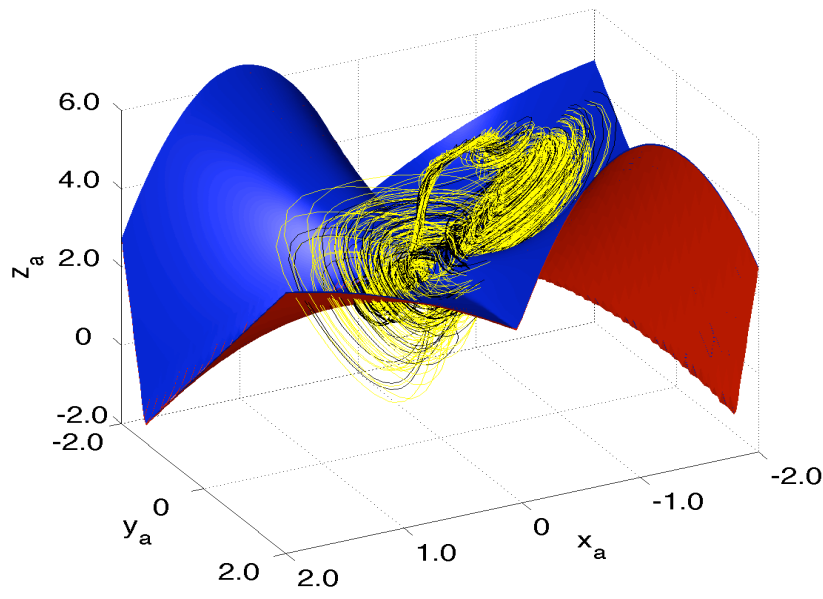

Fig. 7. Surface separating subset $V_{1}$ in phase space and its relative position to the fast subsystem attractor. The surface separates the $V_{1}$ region located above the blue side of the surface where all eigenvalues have negative real parts (convergent), and the region below the red side of the surface corresponds to the region where uncertainties grow exponentially along divergent directions. Yellow lines show the trajectories of the fast model ensemble members and black the ensemble mean.

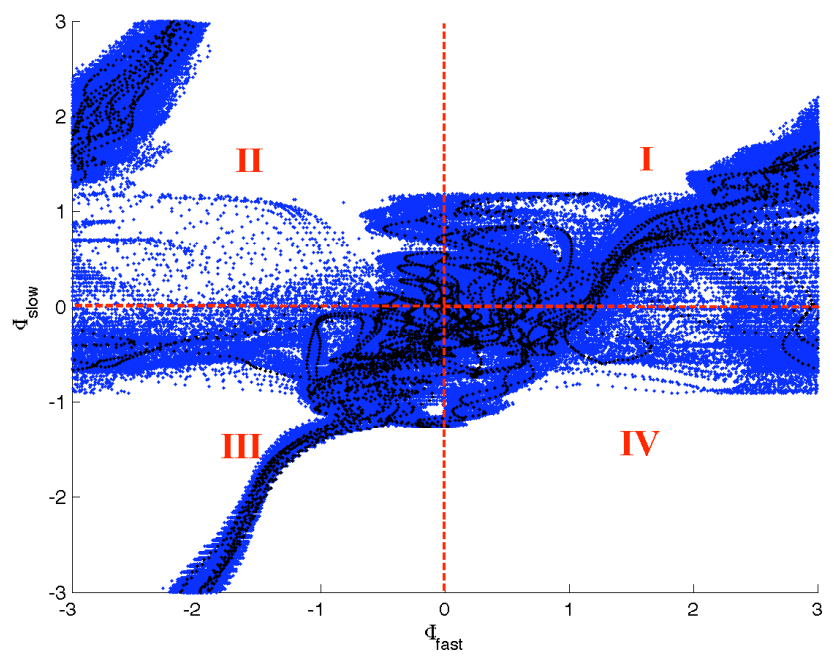

Fig. 8. Phase $\Phi_{\text {slow }}$ (slow model) against $\Phi_{\text {fast }}$ (fast model). Blue dots are ensemble members, black dots ensemble mean.

states the phase pairs are confined into strips in the sub-plane interval since if the subsystems are phase locked then the phase difference $n \Phi_{\text {fast }}-m \Phi_{\text {slow }} \approx$ const, therefore one of the phases can be written as a linear function of the other $\Phi_{\text {slow }}=A+B \Phi_{\text {fast }}$ where $A=-$ const $/ m$ and $B=n / m$. Trajectories are phase locked in regions II and III in Fig. 8.

One can better interpret the motion of the ensemble trajectories on the attractor by combining Fig. 7 and Fig. 8. When a warm event starts to develop in the slow model it forces the ensemble members to cross the boundary into the con- vergent region above the blue side of the surface on the top right of Fig. 7. This can also be seen in Fig. 8 where the ensemble members move to the top right of region I. Figure 8 is periodic on both axis. As the positive anomaly reaches maturity, the ensemble members have already moved way into the convergent region, above the blue side of the surface in Fig. 7, where all eigenvalues are negative. Inside the convergent region all uncertainties start to decay as shown by the passage of ensemble members from region II to III (Fig. 8) and converging ensemble members "spiral" on the top right of Fig. 7. The ensemble trajectories stay long enough inside the convergent subset so that the ensemble becomes locked to the ensemble mean, i.e. internal variability is reduced, so that only the coupled signal is left (Fig. 8 region III and yellow ensemble members "stream" at the center of Fig. 7 flowing from the right side to the left side of Fig. 7). It is worth noting that the time spent by the ensemble members inside the convergent region is dependent on the slowness of the ocean model. Once the ensemble is locked, then it can be pulled away from the convergent region by consistent ocean forcing (slow model moves towards neutral-cold state). The ensemble members cross the boundary going outside the convergent region, then uncertainties grow exponentially along divergent directions (back to the center of Fig. 8 and below the red side of the surface in Fig. 7).

The crossing of ensemble members into the convergent region is not exclusive of warm events since the members quickly cross the boundaries during other periods, as it can be seen from the small fluctuations in spread when the slow model is in "neutral-cold" state (Fig. 6). Making the slow model faster reduces the length of the high predictability periods. For instance, if the slow model time scale is set to $\tau \gtrsim 0.5$ (five times slower than the fast model instead of ten) it implies in the extinction of any high predictability period with only small fluctuations in spread left. Changing the amplitude of the anomalies has a different effect and completely modifies the shape and stability of both attractors.

\section{Conclusions}

The focus of this paper has been to demonstrate that the linear intuition which suggest that uncertainties will steadily increase with lead time can be misleading if the uncertainty dynamics depends strongly on its location in state space, and on the non-normality of the Jacobian matrix. The skewness in the state variables of both subsystems is originated by the forcing through coupling terms projecting along the dominant EOF of each subsystem. In the nonlinear system used in this work the spread of ensemble members is highly dependent on the mean state corresponding to asymmetries in predictability.

The organization of predictability in the Lorenz 63 model has previously been numerically quantified by Nese (1989) through two-dimensional maps showing variations in local 
average divergence rates at several locations on the attractor. Smith et al. (1999) showed the existence of finite volumes of state-space within all perturbations decrease with time for a finite period, stressing the importance of the non-normality of the Jacobian matrix.

For the coupled system here, both the slowness of the slow model, and the intensity of the boundary forcing anomalies contributes to the asymmetry and phase locking of both subsystems. The mechanisms controlling the fast model spread were uncovered revealing uncertainty dynamics depending on the location of ensemble members in the fast model phase space.

One important aspect is that the low order coupled system in this study is simple enough, making it easy to find "convergent regions" of phase space. Within the convergent regions, all eigenvalues of the Jacobian (time dependent propagator) are negative, so that the ensemble members converge. If the members stay long enough within the convergent subsets, depending on the slowness of the slow model, they can synchronize with the slow model forcing. Once both subsystems are phase locked and the fast model internal variability is reduced, the consistent slow model forcing (coupled signal) drives the ensemble out of the convergent subsets. These results suggest that the slow model is indeed reducing the fast model "noise" during some periods where there is higher predictability (reduced spread).

A direct comparison of the results from the simple model employed here with the results from Kirtman et al. (2005) and Wu and Kirtman (2006) cannot be made since the model employed here is distinctly too simple to describe the detailed physics of a realistic climate system. Although the generalization among different nonlinear systems is always difficult since the predictability of evolving uncertainties is a model intrinsic quantity, the results presented here should be of use in predictability studies of more complex systems like state-of-the-art CGCMs. A pertinent question for future directions bears upon whether these findings are true for larger and more complex systems and, in particular, for CGCMs, or whether these results have solely uncovered properties of a low-order coupled model.

\section{Appendix A}

\section{Lorenz Coupled Fast-Slow operators}

For the Coupled Lorenz 63 model the uncoupled nonlinear operator

$$
U_{\mathbf{X}}=\left(\begin{array}{ccccccc}
-\sigma & \sigma & 0 & & & \\
r & -1 & -x_{a} & & 0 & \\
0 & x_{a} & -b & & & \\
& & & -\tau \sigma & \tau \sigma & 0 \\
& 0 & & \tau r & -\tau & -\tau x_{o} \\
& & & 0 & \tau x_{o} & -\tau b
\end{array}\right)
$$

and the coupled linear operator:

$$
C=\left(\begin{array}{cccccc} 
& \multicolumn{1}{c}{-\alpha} & 0 & 0 \\
& & & 0 & \alpha & 0 \\
& & & 0 & 0 & \alpha \\
-\alpha & 0 & 0 & & & \\
0 & \alpha & 0 & & 0 & \\
0 & 0 & -\alpha & &
\end{array}\right) .
$$

The Jacobian of the uncoupled operator is given by:

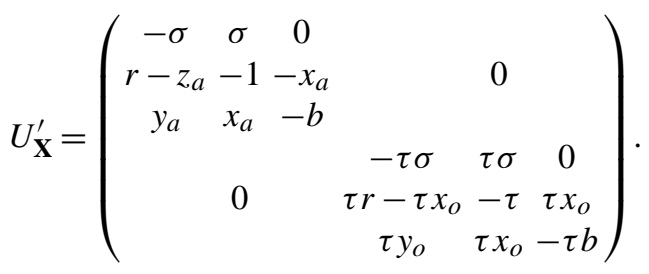

\section{A1 Routh-Hurwitz Criteria and Convergent Subsets}

The Routh-Hurwitz Criteria give necessary and sufficient conditions for all the roots of the characteristic polynomial with real coefficients to lie in the left half of the complex plane. It states that, given a characteristic polynomial

$P(\lambda)=\lambda^{n}+a_{1} \lambda^{n-1}+\ldots+a_{n-1} \lambda+a_{n}$

where the coefficients $a_{i}$ are real constants, with $i=1, \ldots, n$, define the Hurwitz matrices using the coefficients $a_{i}$ of the characteristic polynomial

$$
H_{1}=\left(a_{1}\right), \quad H_{2}=\left|\begin{array}{cc}
a_{1} & 1 \\
a_{3} & a_{2}
\end{array}\right| \text { and } H_{3}=\left|\begin{array}{ccc}
a_{1} & 1 & 0 \\
a_{3} & a_{2} & a_{1} \\
a_{5} & a_{4} & a_{3}
\end{array}\right|
$$

and:

$$
H_{n}=\left|\begin{array}{cccccc}
a_{1} & 1 & 0 & 0 & \ldots & 0 \\
a_{3} & a_{2} & a_{1} & 1 & \ldots & 0 \\
a_{5} & a_{4} & a_{3} & a_{2} & \ldots & 0 \\
\vdots & \vdots & \vdots & \vdots & \ldots & 0 \\
0 & 0 & 0 & 0 & \ldots & a_{n}
\end{array}\right|
$$

All the roots of $P(\lambda)$ are negative or have negative real part if and only if the determinants of all Hurwitz matrices are positive:

$$
\operatorname{det} \quad H_{j}>0, \quad j=1,2, \ldots, n .
$$

\section{Coupled Lorenz Model Convergent Subsets}

For the coupled Lorenz model the coefficients of the characteristic polynomial are $a_{1}=\sigma+1+b, \quad a_{2}=\sigma(1+b-r+$ $\left.z_{a}\right)+b+x_{a}^{2}$, and $a_{3}=\sigma\left(1-r b+x_{a}^{2}+b z_{a}+x_{a} y_{a}\right)$. Given the parameters $\sigma, b, r>0$, then $H_{0}=1>0$ and $H_{1}=a_{1}=$ $\sigma+1+b>0$. Therefore, we only need to find the conditions for $H_{2}=a_{1} a_{2}-a_{3}$ to be positive

$$
\begin{aligned}
\left\{\mathbf{X}^{a} \mid H_{2}>0\right\}=\left\{\mathbf{X}^{a} \mid z_{a}\right. & >z_{1}\left(x_{a}, y_{a}\right) \\
& \left.=b_{1}+b_{2} x_{a}^{2}+b_{3} x_{a} y_{a}\right\}
\end{aligned}
$$


where

$$
\begin{aligned}
& b_{1}=\sigma_{2} r+\sigma r-\sigma^{2}-\sigma^{2} b-2 \sigma b-b-\sigma b^{2}-b^{2} \sigma \\
& b_{2}=-\frac{1+b}{\sigma^{2}+\sigma} \\
& b_{3}=\frac{1}{1+\sigma} \\
& \left\{\begin{aligned}
\left\{\mathbf{X}^{a} \mid a_{3}>0\right\}=\left\{\mathbf{X}^{a} \mid z_{a}\right. & >z_{2}\left(x_{a}, y_{a}\right) \\
& \left.=c_{1}+c_{2} x_{a}^{2}+c_{3} x_{a} y_{a}\right\}
\end{aligned}\right.
\end{aligned}
$$

with $c_{1}=r-1$, and $c_{2}=c_{3}=-\frac{1}{b}$ leading to:

$$
\begin{aligned}
V_{1}=\left\{\mathbf{X}^{a} \quad\right. & \left.H_{0}, H_{1}, H_{2}, a_{3}>0\right\} \\
= & \left\{\mathbf{X}^{a} \mid z_{a}>z^{*}=\max \left(z_{1}, z_{2}\right)\right\} .
\end{aligned}
$$

The determinants of the three principal submatrices of $\left[-\left(A_{\mathbf{X}^{a}}^{\prime}+A_{\mathbf{X}^{a}}^{\prime T}\right)\right]$ are:

$$
\begin{aligned}
& D_{1}=2 \sigma \\
& D_{2}=4 \sigma-\left(\sigma+r-z_{a}\right)^{2} \\
& D_{3}=2 b\left[4 \sigma-\left(\sigma+r-z_{a}\right)^{2}\right]-2 y_{a}^{2} .
\end{aligned}
$$

All states $\mathbf{X}^{a}$ within an elliptical tube build the subset of states with negative eigenvalues of $\left(A_{\mathbf{X}^{a}}^{\prime}+A_{\mathbf{X}^{a}}^{\prime}\right)$ :

$$
\begin{aligned}
V_{2}=\left\{\mathbf{X}^{a}\right. & \left.\mid D_{1}, D_{2}, D_{3}>0\right\} \\
= & \left\{\mathbf{X}^{a} \mid \frac{\left(z_{a}-(r+\sigma)\right)^{2}}{4 \sigma}+\frac{y_{a}^{2}}{4 b \sigma}<1\right\} .
\end{aligned}
$$

Acknowledgements. The authors acknowledge support from NSF grants OCI0749165, ATM0754341, NOAA grant NA08OAR432088 and CAPES/FULBRIGHT (BEX2837/064).

Edited by: J. Duan

Reviewed by: A. Tsonis and three other anonymous referees

\section{References}

Boffetta, G., Crisanti, A., Paparella, F., Provenzale, A., and Vulpiani, A.: Slow and fast dynamics in coupled systems: A time series analysis view, Physica D, 116, 301-312, doi:10.1016/S01672789(97)00300-X, 1998.

Duane, G. and Tribbia, J. J.: Nonlinear Dynamics in Geosciences, chap. Dynamical synchronization of truth and model as an approach to data assimilation, parameter estimation, and model learning, 291-310, Springer, NY, doi:10.1007/978-0-387-349183-17, 2007.
Frankignoul, C. and Hasselman, K.: Stochastic climate models, Part II Application to sea-surface temperature anomalies thermocline variability, Tellus, 29, 289-305, 1977.

Hasselman, K.: Stochastic climate models, Part I, Theory, Tellus, 28, 473-485, 1976.

Kirtman, B. and Shukla, J.: Interactive coupled ensemble: A new coupling strategy for GCMs, Geophys. Res. Lett., 29, 10291032, 2002.

Kirtman, B., Pegion, K., and Kinter, S. M.: Internal Atmospheric Dynamics and Tropical Indo-Pacific Climate Variability, J. Atmos. Sci., 62, 2220-2233, 2005.

Lorenz, E. N.: Deterministic Nonperiodic Flow, J. Atmos. Sci., 20, 130-141, 1963.

Miller, R. N., Ghil, M., and Gauthiez, F.: Advanced Data Assimilation in Strongly Nonlinear Dynamical Systems, J. Atmos. Sci., 51, 1037-1056, 1994.

Kumar Mittal, A., Dwivedi, S., and Chandra Pandey, A.: Bifurcation analysis of a paradigmatic model of monsoon prediction, Nonlin. Processes Geophys., 12, 707-715, doi:10.5194/npg-12707-2005, 2005.

Nese, J. M.: Quantifying local predictability in phase space, Physica, 35D, 237-250, 1989.

Palmer, T. N.: Extended-Range Atmospheric Prediction and the Lorenz Model, B. Am. Meteorol. Soc., 74, 49-66, 1993.

Peña, M. and Kalnay, E.: Separating fast and slow modes in coupled chaotic systems, Nonlin. Processes Geophys., 11, 319-327, doi:10.5194/npg-11-319-2004, 2004.

Rosenblum, M., Pikovsky, A., and Kurths, J.: Phase synchronization of chaotic oscillators, Phys. Rev. Lett, 76, 1804-1996, 1996.

Shukla, J., Anderson, J., Baumhefner, D., Brankovic, C., Chang, Y., Kalnay, E., Marx, L., Palmer, T., Paolino, D., Ploshay, J., Schubert, S., Straus, D., Suarez, M., and Tribbia, J.: Dynamical Seasonal Prediction, B. Am. Meteorol. Soc., 81, 2593-2606, 2000.

Smith, L. A., Ziehmann, C., and Fraedich, K.: Uncertainty dynamics and predictabiity in Chaotic systems, Q. J. Roy. Meteor. Soc. 125, 2855-2886, 1999.

Sura, P., Newman, M., Penland, C., and Sardeshmukh, P.: Multiplicative Noise and Non-Gaussianity: A Paradigm for Atmospheric Regimes?, J. Atmos. Sci., 62, 2005.

Tsonis, A. A.: Chaos: from theory to applications, Plenum, NY, 274 pp., 1992.

Wittenberg, A. T. and Anderson, J. L.: Dynamical implications of prescribing part of a coupled system: Results from a low-order model, Nonlin. Processes Geophys., 5, 167-179, doi:10.5194/npg-5-167-1998, 1998.

$\mathrm{Wu}, \mathrm{R}$. and Kirtman, B. P.: Changes in spread and predictability associated with ENSO in an ensemble coupled GCM, J. Climate, 19, 4378-4396, 2006.

Zebiak, S. E. and Cane, M. A.: A model of El Niño Southern Oscillation, Mon. Wea.. Rev., 115, 2262-2278, 1987. 